\title{
Direct and sequential four-body recombination rates at low temperatures
}

\author{
E. Garrido ${ }^{*}{ }^{*}$ \\ Instituto de Estructura de la Materia, IEM-CSIC, Serrano 123, E-28006 Madrid, Spain \\ A. S. Jensen \\ Department of Physics and Astronomy, Aarhus University, DK-8000 Aarhus C, Denmark
}

(Received 23 March 2021; accepted 10 May 2021; published 24 May 2021)

\begin{abstract}
We investigate four-body nuclear reactions in stellar environments contributing to the creation of light nuclei, exemplified by ${ }^{9} \mathrm{Be}$ and ${ }^{12} \mathrm{C}$. The originally assumed process is radiative capture, where nuclear clusters combine into the excited final nucleus and photon emission populates the stable nuclear ground states. Instead, we consider nuclear four-body recombination reactions where a spectator nuclear particle replaces the photon. We first develop the elaborate formalism for both direct and sequential capture processes, where the decaying threebody resonance is formed without and with population of an intermediate two-body resonance, respectively. To facilitate both calculations and practical applications we parametrize the involved cross sections as done successfully in previous computations of reaction rates. We consider the lowest-lying nuclear states with their dominant contributions at low stellar temperatures. We calculate and compare reaction and production rates for different processes. The direct reaction mechanism dominates by many orders of magnitude at low temperature, where the sequential stepping stones are energetically too expensive to use. At somewhat higher temperatures these two different nuclear four-body mechanisms become comparable. Comparison to radiative three-body capture reveals already formally, but also numerically, that four-body nuclear recombination must dominate for sufficiently high nuclear densities. Numerical values are given for all these rates as a function of temperature and density. The relative importance is exhibited.
\end{abstract}

DOI: 10.1103/PhysRevC.103.055813

\section{INTRODUCTION}

The spontaneous radiative capture of three $\alpha$ particles to produce ${ }^{12} \mathrm{C}$ is known to play a crucial role in order to bridge the $A=5$ and $A=8$ gaps in the nuclear chart [1]. This is a three-body reaction that becomes relevant in high density scenarios due to the absence of alternative simpler routes to the ${ }^{12} \mathrm{C}$ production. This process is usually treated assuming a sequential mechanism [2], i.e., as two consecutive two-body processes, where in a first step the $0^{+}$particle-unstable state in ${ }^{8} \mathrm{Be}$ is populated. The small width of this resonance permits, in the second step, to capture a third $\alpha$ particle and form ${ }^{12} \mathrm{C}$ before decaying of the ${ }^{8} \mathrm{Be}$ resonance. The width of the two-body resonance, even if very small, is still playing an important role, as argued in Refs. [3,4], where it is shown that for sufficiently low temperatures $\left(T \lesssim 3 \times 10^{7} \mathrm{~K}\right)$ the capture proceeds through the low energy wing of the $\alpha-\alpha$ resonance, which gives rise to a significant increase of the reaction rate. Furthermore, in Ref. [5] it has been shown that a direct capture mechanism, without population of intermediate two-body states, could also be crucial at very low temperatures, where the particle energy is still too small to populate the intermediate state. The direct mechanism could actually be responsible for an increase of several orders of magnitude of the reaction rate in the low-temperature region.

\footnotetext{
*e.garrido@csic.es
}

Together with radiative capture processes, it has been suggested in Ref. [6] that pure nuclear interactions could also play an important role in the de-excitation of ${ }^{12} \mathrm{C}$ excited states. Inelastic collisions with surrounding neutrons, protons, $\alpha$ particles, and perhaps other ions, were estimated to substantially increase the rate of formation of ${ }^{12} \mathrm{C}$ for neutron densities above $10^{7} \mathrm{~g} / \mathrm{cm}^{3}$ and temperatures greater than a few GK. Preliminary studies of the reaction rate enhancement produced by inelastic $\alpha$ and proton scattering were reported in Refs. $[7,8]$.

Recent calculations have corroborated these conclusions. In Refs. $[9,10]$ the possibility of four-body recombination, or particle induced de-excitation (both denominations will be indistinctly used in this work), was pointed out as an alternative mechanism to the spontaneous three-body radiative decay. For densities higher than $10^{6} \mathrm{~g} / \mathrm{cm}^{3}$ the four-body recombination dominates, especially for temperatures below $1 \mathrm{GK}$.

This sort of particle-induced reaction rate enhancement has recently been revisited [11]. The theoretical background behind this work is as in Refs. [6-8], but considering also the possibility of the neutron as the particle that boosts the decay. The main difference is that in Ref. [11] a more sophisticated procedure in order to obtain the crucial $\alpha$, proton, and neutron inelastic cross sections on ${ }^{12} \mathrm{C}$ has been employed. Again, a relevant enhancement of the reaction rate was found for sufficiently large temperatures and densities.

In these works [6-8], and also in the recent paper [11], the reaction rate was obtained by describing the process as 
a simple two-body process, where a previously populated three-body resonance, due to the presence in the environment of neutrons, protons, or $\alpha$ particles, decays into a three-body bound state, such that the excess of energy is transformed into kinetic energy of the fourth particle. Thanks to the detailed balance principle, this process can be easily related to the inverse reaction, namely the inelastic excitation of the bound three-body state by the fourth particle, either a neutron, or a proton, or an $\alpha$ particle. The available experimental information or the reliability of the theoretical calculation of these inelastic cross sections becomes then crucial in order to obtain also reliable estimates of the reaction rates.

This strong simplification (to a two-body process) of what really is a four-body process amounts to ignoring the fact that the decaying three-body resonance actually has a finite width, and therefore a finite lifetime, treating in practice the resonance as a bound state with an energy equal to the resonance energy. However, it is known that for radiative decay the finite width of the resonance determines to a very large extent the value of the reaction rate, especially a low temperatures [2]. Furthermore, not only the width, but also the reaction mechanism, through which the resonance is populated, plays an important role. In Ref. [5] it is shown how, for temperatures about $0.01 \mathrm{GK}$, a direct population of the resonance could lead to radiative reaction rates, for formation of ${ }^{12} \mathrm{C}$ or ${ }^{9} \mathrm{Be}$, of up to seven orders of magnitude bigger than by the sequential mechanism, where the intermediate low-lying $0^{+}$resonance in ${ }^{8} \mathrm{Be}$ is populated before capturing the third particle.

These two elements, the finite resonance width, and the reaction mechanism, should also play an important role in the case of four-body recombination reactions, and they could therefore also be relevant when comparing to the radiative process and the circumstances under which the particleinduced reaction rate is dominating. The main goal of this work is therefore to investigate the effect of these two ingredients, resonance width and reaction mechanism, in the four-body recombination reaction rates, and check how these rates compete with the ones corresponding to a spontaneous radiative process. The cases of the formation of ${ }^{9} \mathrm{Be}$ and ${ }^{12} \mathrm{C}$ induced by the presence of protons, neutrons, or $\alpha$ particles will be considered.

This work follows a scheme similar to the one in Ref. [5], where the radiative capture process leading to ${ }^{9} \mathrm{Be}$ and ${ }^{12} \mathrm{C}$ was investigated. We first give the general expressions for the four-body recombination reaction rate. The analytic expressions corresponding to a direct and a sequential capture mechanism are derived in Secs. III and IV, respectively. For each of these two cases, the expressions for the limit of zero width for the resonances are also derived. In order to facilitate the reading of the paper and avoid an unnecessary rereading of Secs. III and IV, we summarize in Sec. V the key equations relevant for the subsequent calculations assuming either direct or sequential capture mechanism. Section V is closed with some remarks concerning the production rates and the comparison with the rate corresponding to the radiative capture process. The numerical results are presented in details in Sec. VI for the important cases of ${ }^{9} \mathrm{Be}$ and ${ }^{12} \mathrm{C}$. We close the paper with the summary and conclusions presented in Sec. VII.

\section{THE FOUR-BODY NUCLEAR RECOMBINATION RATE}

The goal in this work is to investigate the four-body recombination rate for a given reaction $a+b+c+d \rightarrow A+d$, where $A$ is a bound state formed by particles $a, b$, and $c$. In general, particles $a, b$, and $c$ will be assumed to populate a three-body resonance, which will be in fact the one decaying into the bound state $A$. Particle $d$ is simply acting as a mere spectator, and its only role is to maintain energy and momentum in the resonance decay process. Reactions where particle $d$ is exchanged with one of the ones forming the three-body resonance will not be considered here.

Let us denote the initial four-body energy as $E$. Therefore, by energy conservation we must have that $E=T_{A d}^{\prime}+B=$ $T_{A d}^{\prime}-|B|$, where $B$ is the binding energy of the three-body system $A$, and $T_{A d}^{\prime}$ is the final relative kinetic energy between $A$ and $d$. In Ref. [10] it is shown that the general expression for the four-body reaction rate, averaged over the four-body Maxwellian energy distribution, takes the following form as a function of the temperature $T$ :

$$
\begin{aligned}
\left\langle R_{a b c d}(E)\right\rangle= & \frac{4(2 \pi)^{\frac{5}{2}} \hbar^{6} \mu_{A, d}}{\left(\mu_{a, b} \mu_{a b, c} \mu_{a b c, d}\right)^{3 / 2}} \frac{g_{A}}{g_{a} g_{b} g_{c}} \\
& \times \frac{1}{(K T)^{\frac{9}{2}}} \int_{0}^{\infty} T_{A d}^{\prime} \sigma_{A d}\left(T_{A d}^{\prime}\right) e^{-\frac{E}{K T}} d E,
\end{aligned}
$$

where $K$ is the Boltzmann constant, $\sigma_{A d}$ is the cross section for the breakup reaction $A+d \rightarrow a+b+c+d$, and $\mu_{i, j}$ represents the reduced mass of particle $j$ and particle (or group of particles) $i$.

The cross section $\sigma_{A d}$ is related to the capture cross section $\sigma_{a b c d}$ of the inverse process by means of the principle of detailed balance. Both cross sections contain the same matrix element, $\left\langle\Psi_{i}|W| \Psi_{f}\right\rangle$, where $\Psi_{i}$ and $\Psi_{f}$ are the initial and final four-body wave functions, and $W$ represents the interaction responsible for the reaction. Therefore, calculation of the reaction rate in Eq. (1) requires in principle knowledge of the two four-body wave functions, one of them representing the four particles in the continuum, and the other one describing a bound three-body state plus the fourth particle in the continuum.

Therefore, calculation of Eq. (1) is in general a rather complicated task, which, unless some simplifications, like in Ref. [10], are made, unavoidably requires a calculation of four-body wave functions. Furthermore, even if some simplifications are introduced, the competition between the direct and sequential mechanism is hidden by the procedure itself. It would be rather difficult to extract from the structure of the four-body states which of the two mechanisms has actually been predominantly used.

For this reason we consider it useful to perform the same kind of analysis for the four-body recombination rate as made in Ref. [5] for the three-body radiative capture process. The idea is to compare the direct and sequential capture mechanisms in the four-body recombination reaction rates using realistic phenomenological parametrizations of the cross sections entering in the reactions. In this way it will be possible to study in a clear way the effects arising from the different positions and widths of the two- and three-body resonances 
involved, as well as the effects produced by these two capture mechanisms.

As we will see, the analytical expressions describing the process under investigation will contain two different types of cross sections. The first type corresponds to capture processes leading to a two-body or a three-body resonance, which will be described as simple Breit-Wigner shaped cross sections, depending on the energy and width of the populated resonance. The second type is the cross section $\sigma_{A d}$, present also in Eq. (1), but now understood as an inelastic two-body process, where the system $A$ is just excited after the collision with particle $d$. Following [2,12], when $A$ and $d$ are both charged, the inelastic cross section takes the general form

$$
\sigma_{A d}\left(T_{A d}^{\prime}\right)=\frac{S\left(T_{A d}^{\prime}\right)}{T_{A d}^{\prime}} \exp (-2 \pi \eta)
$$

with $\eta=Z_{A} Z_{d} e^{2} /(\hbar v)$, where $e$ is the electron charge, $Z_{A}$ and $Z_{d}$ are the charges of the two particles, and $v$ their relative velocity. If either $A$ or $d$ is not charged we take instead [12]

$$
\sigma_{A d}\left(T_{A d}^{\prime}\right)=\frac{S\left(T_{A d}^{\prime}\right)}{\sqrt{T_{A d}^{\prime}}} .
$$

The $S$ factor $S\left(T_{A d}^{\prime}\right)$ is typically taken as an expansion in some powers of the energy, whose expansion parameters are adjusted to reproduce the available experimental information concerning the cross section.

\section{DIRECT CAPTURE MECHANISM}

Let us consider the case where the three-body resonance is formed directly, without population of any intermediate twobody state. We first developed the general formalism, which afterwards is employed with practical parametrizations.

\section{A. Computational scheme}

The recombination process under consideration takes place in two steps:

$$
\underbrace{a+b+c+d}_{E} \rightarrow \underbrace{(a b c)}_{E^{\prime \prime}}+d \rightarrow \underbrace{A+d}_{T_{A d}^{\prime}+B}
$$

where the first step is a three-body process where the resonance $(a b c)$ is populated, and the second one is a two-body process where the resonance $+d$ system decays into $A+d$.

Let us denote here by $E$ the total four-body energy, by $E^{\prime}$ the relative energy between particle $d$ and the center of mass of the $(a b c)$ system, and by $E^{\prime \prime}$ the three-body energy of the $(a b c)$ system. It is then clear that

$$
E=E^{\prime}+E^{\prime \prime}=T_{A d}^{\prime}+B=T_{A d}^{\prime}-|B|,
$$

where, as before, $B$ is the binding energy of the three-body system $A$, and $T_{A d}^{\prime}$ is the final relative kinetic energy between $A$ and $d$.
The reaction rate corresponding to the final two-body step can be found for instance in Eq. (32) of Ref. [12]:

$$
\begin{aligned}
\left\langle R_{a b c, d}\left(E^{\prime \prime}\right)\right\rangle= & \sqrt{\frac{8}{\pi}} \frac{1}{\mu_{a b c, d}^{1 / 2}} \frac{1}{(K T)^{3 / 2}} \\
& \times \int_{0}^{\infty} E^{\prime} \sigma_{a b c, d}\left(E^{\prime}, E^{\prime \prime}\right) e^{-\frac{E^{\prime}}{K T}} d E^{\prime},
\end{aligned}
$$

where $K$ again is the Boltzmann constant, $T$ is the temperature, and $\sigma_{a b c, d}\left(E^{\prime}, E^{\prime \prime}\right)$ is the two-body cross section for the process $(a b c)+d \rightarrow A+d$.

In order to get the total reaction rate one has to multiply Eq. (5) by the formation probability of the three-body $(a b c)$ resonance, and average with the Maxwell-Boltzmann distribution for three particles:

$$
B_{3}\left(E^{\prime \prime}, T\right)=\frac{1}{2} \frac{E^{\prime \prime 2}}{(K T)^{3}} e^{-\frac{E^{\prime \prime}}{K T}} .
$$

The population probability for the three-body resonance is given by the product of the resonance lifetime, $\tau_{a b c}=\hbar / \Gamma_{a b c}$, and the rate of formation of the resonance. This rate is given by the flux of particles times the three-body cross section, $\sigma_{a b c}\left(E^{\prime \prime}\right)$. As shown in Ref. [13], the flux of particles for three-body collisions is given by

$$
\text { flux }=\frac{\hbar \kappa^{\prime \prime}}{m}\left(\frac{m}{\mu_{a, b}}\right)^{3 / 2}\left(\frac{m}{\mu_{a b, c}}\right)^{3 / 2},
$$

where $\kappa^{\prime \prime 2}=2 m E^{\prime \prime} / \hbar^{2}$, and $m$ is the usual normalization mass introduced to construct the Jacobi coordinates commonly used to describe three-body systems. It is clear that the flux written above is ill-defined, since it depends on the arbitrary choice of the normalization mass. However, as we shall show later, the dependence on $m$ disappears in the final expression of the reaction rate. Therefore, we get for the total reaction rate

$$
\begin{aligned}
\left\langle R_{a b c d}\right\rangle= & \left(\frac{m}{\mu_{a, b}}\right)^{3 / 2}\left(\frac{m}{\mu_{a b, c}}\right)^{3 / 2} \\
& \times \int d E^{\prime \prime} B_{3}\left(E^{\prime \prime}, T\right) \frac{\hbar}{\Gamma_{a b c}} \frac{\hbar \kappa^{\prime \prime}}{m} \sigma_{a b c}\left(E^{\prime \prime}\right)\left\langle R_{a b c, d}\left(E^{\prime \prime}\right)\right\rangle,
\end{aligned}
$$

which by use of Eqs. (5) and (6) leads to

$$
\begin{aligned}
\left\langle R_{a b c d}\right\rangle= & \frac{2 \hbar}{\sqrt{\pi}} \frac{m^{5 / 2}}{\left(\mu_{a, b} \mu_{a b, c}\right)^{3 / 2} \mu_{a b c, d}^{1 / 2}} \frac{1}{(K T)^{9 / 2}} \\
& \times \int d E^{\prime \prime} E^{\prime \prime 5 / 2} \frac{\sigma_{a b c}\left(E^{\prime \prime}\right)}{\Gamma_{a b c}} e^{-\frac{E^{\prime \prime}}{K T}} \\
& \times \int d E^{\prime} E^{\prime} \sigma_{a b c, d}\left(E^{\prime}, E^{\prime \prime}\right) e^{-\frac{E^{\prime}}{K T}} .
\end{aligned}
$$

Finally, we can now make use of the detailed balance principle that relates $\sigma_{a b c, d}$ with the cross section $\sigma_{A d}$ for the inverse process, which is

$$
\sigma_{a b c, d}=\frac{g_{A} g_{d}}{g_{a b c} g_{d}} \frac{\mu_{A, d}}{\mu_{a b c, d}} \frac{T_{A d}^{\prime}}{E^{\prime}} \sigma_{A d},
$$

where $g_{i}=\left(2 J_{i}+1\right)$ is the multiplicity of system $i$ with angular momentum $J_{i}$. Therefore, it is possible to rewrite Eq. (9) 
in terms of $\sigma_{A d}$ (in principle accessible experimentally) and get the final expression for the total reaction rate assuming a direct formation of the three-body resonance:

$$
\begin{aligned}
\left\langle R_{a b c d}\right\rangle= & \frac{g_{A}}{g_{a b c}} \frac{2 \hbar}{\sqrt{\pi}} \frac{m^{5 / 2} \mu_{A, d}}{\left(\mu_{a, b} \mu_{a b, c} \mu_{a b c, d}\right)^{3 / 2}} \frac{1}{(K T)^{9 / 2}} \\
& \times \int d E^{\prime \prime} E^{\prime \prime 5 / 2} \frac{\sigma_{a b c}\left(E^{\prime \prime}\right)}{\Gamma_{a b c}} e^{-\frac{E^{\prime \prime}}{K T}} \\
& \times \int d E^{\prime} T_{A d}^{\prime} \sigma_{A d}\left(T_{A d}^{\prime}\right) e^{-\frac{E^{\prime}}{K T}} .
\end{aligned}
$$

All along the derivations, we have not considered the possibility of having identical particles involved in the process. In order to do so, the expression above should be multiplied by $\prod_{i} v_{i}$ !, where $v_{i}$ is the number of identical particles of type $i$.

\section{B. Three-body resonant cross section}

In the reaction rate (11), together with $\sigma_{A d}$, the key quantity is the three-body resonant cross section $\sigma_{a b c}\left(E^{\prime \prime}\right)$, which is given by [14]

$$
\sigma_{a b c}\left(E^{\prime \prime}\right)=\frac{32 \pi^{2}}{\kappa^{\prime \prime 5}} \frac{g_{a b c}}{g_{a} g_{b} g_{c}} \frac{\Gamma_{a b c}^{2}}{\left(E^{\prime \prime}-E_{a b c}\right)^{2}+\Gamma_{a b c}^{2} / 4},
$$

where $E_{a b c}$ is the energy of the three-body resonance. Since $\kappa^{\prime \prime}=\sqrt{2 m E^{\prime \prime}} / \hbar$, we now see that inclusion of Eq. (12) into Eq. (11) eliminates the dependence on the arbitrary normalization mass $m$.

In general, the width $\Gamma_{a b c}$ will depend on the three-body energy $E^{\prime \prime}$, typically as [15]

$$
\Gamma_{a b c}\left(E^{\prime \prime}\right)=\Gamma_{a b c}^{(0)} \frac{P\left(E^{\prime \prime}\right)}{P\left(E_{a b c}\right)},
$$

where $P\left(E^{\prime \prime}\right)$ is the penetration factor.

For direct decay of the three-body resonance, and for the case of charged particles, as shown in Eqs. (3) and (12) of [15], we take

$$
\Gamma_{a b c}\left(E^{\prime \prime}\right)=\Gamma_{a b c}^{(0)} \frac{1+e^{2 b_{a b c}} / \sqrt{E_{a b c}}}{1+e^{2 b_{a b c} / \sqrt{E^{\prime \prime}}}}
$$

with

$$
b_{a b c}=\frac{\pi}{2} \sqrt{\frac{2}{\hbar^{2}\left(m_{a}+m_{b}+m_{c}\right)}}\left(\sum\left(Z_{i} Z_{j} e^{2}\right)^{2 / 3}\left(m_{i} m_{j}\right)^{1 / 3}\right)^{3 / 2},
$$

where $m_{a}, m_{b}$, and $m_{c}$ are the masses of the particles, $Z_{i}$ is the charge of particle $i$, and the sum runs over the three possible pairs of particles.

Finally, let us consider the extreme case, where the width of the three-body resonance is neglected and taken equal to zero. In this case we then have that

$$
\frac{\sigma_{a b c}\left(E^{\prime \prime}\right)}{\Gamma_{a b c}} \stackrel{\Gamma_{a b c} \rightarrow 0}{\longrightarrow} \frac{64 \pi^{3}}{\kappa_{a b c}^{5}} \frac{g_{a b c}}{g_{a} g_{b} g_{c}} \delta\left(E^{\prime \prime}-E_{a b c}\right),
$$

where $\kappa_{a b c}=\sqrt{2 m E_{a b c}} / \hbar$, which leads to the zero-width limit of the reaction rate:

$$
\begin{aligned}
\left\langle R_{a b c d}\right\rangle= & \frac{g_{A}}{g_{a} g_{b} g_{c}} \frac{4(2 \pi)^{\frac{5}{2}} \hbar^{6} \mu_{A, d}}{\left(\mu_{a, b} \mu_{a b, c} \mu_{a b c, d}\right)^{3 / 2}} \frac{1}{(K T)^{9 / 2}} \\
& \times e^{-\frac{E_{a b c}}{K T}} \int d E^{\prime} T_{A d}^{\prime} \sigma_{A d}\left(T_{A d}^{\prime}\right) e^{-\frac{E^{\prime}}{K T}},
\end{aligned}
$$

which again should be multiplied by $\prod_{i} v_{i} !\left(v_{i}\right.$ is the number of identical particles of type $i$ ) in order to account for the presence of identical particles.

Note that in this limit we have that $E=E^{\prime}+E_{a b c}$, and therefore Eq. (17) is formally identical to Eq. (1), which was presented as the general form of the four-body recombination rate. However, as discussed, in Eq. (1) $\sigma_{A d}$ describes the full four-body process, and contains the intrinsic kinematics of the four particles all along the process for all four-body energies, including the $0 \leqslant E \leqslant E_{a b c}$ low-energy range. On the contrary, in the picture described in this section, the cross section $\sigma_{A d}$ is an inelastic two-body process where the nucleus $A$ is excited into the state with energy $E_{a b c}$, which implies that in the extreme limit that led to Eq. (17) only four-body energies satisfying $E \geqslant E_{a b c}$ are allowed. A description of the reaction rate in the $0 \leqslant E \leqslant E_{a b c}$ energy range requires use of Eq. (11), being the resonant cross section $\sigma_{a b c}\left(E^{\prime \prime}\right)$ the one dictating the low-energy behavior of the reaction rate.

\section{SEQUENTIAL CAPTURE MECHANISM}

The second possibility to be considered is the case of a sequential mechanism, where, prior to the formation of a three-body resonance, an intermediate two-body resonance is populated. We first provide the formulation for the combined processes, and afterwards we describe the individual parametrizations.

\section{A. The combined sequence of reactions}

Several steps are involved in this reaction mechanism. The processes are

$$
\underbrace{a+b+c+d}_{E} \rightarrow \underbrace{(a b)}_{E^{\prime \prime \prime}}+c+d \rightarrow(\underbrace{(a b}_{E_{E^{\prime \prime \prime}}^{(a b)}})+d \rightarrow \underbrace{A+d}_{E^{\prime}},
$$

which are three consecutive two-body processes: The population of the two-body $(a b)$ resonance, the capture of particle $c$ by the two-body $(a b)$ system in order to form the three-body resonance $(a b c)$, and the decay of the resonance into the three-body bound state $A$, whose energy excess is absorbed by particle $d$.

In this case the energy $E^{\prime \prime \prime}$ is the relative energy between particles $a$ and $b$, and $E^{\prime \prime}$ is the relative energy between particle $c$ and the center of mass of the $(a b)$ system (therefore, $E^{\prime \prime}+E^{\prime \prime \prime}$ is the total three-body energy). As before, $E^{\prime}$ is the relative energy between $d$ and the center of mass of the three-body system, and $T_{A d}^{\prime}$ is the final relative energy between $d$ and the bound system $A$. Therefore the energy conservation 
reads now

$$
E=E^{\prime}+E^{\prime \prime}+E^{\prime \prime \prime}=T_{A d}^{\prime}+B=T_{A d}^{\prime}-|B| .
$$

The last two-body step is the same as for the direct capture. Therefore, the reaction rate for this last process is again given by Eq. (5), although now $\sigma_{a b c, d}$ depends on the three energies $E^{\prime}, E^{\prime \prime}$, and $E^{\prime \prime \prime}$. Again, this rate has to be multiplied by the probability for the formation of the three-body resonance. The difference is that this probability is now given by the probability of formation of the two-body $(a b)$ resonance, times the probability of the subsequent capture of particle $c$ in order to form the three-body resonance. Each of these two probabilities is again the product of the resonance lifetime $\tau=\hbar / \Gamma$, the flux of particles $\hbar k / \mu$, and the corresponding cross section. Each of them has also to be averaged by means of the two-body Maxwell-Boltzmann distribution

$$
B_{2}(E, T)=\frac{2}{\sqrt{\pi}} \frac{E^{1 / 2}}{(K T)^{3 / 2}} e^{-\frac{E}{K T}} .
$$

All this leads to the following expression for the total four-body recombination rate in case of a sequential capture mechanism:

$$
\begin{aligned}
\left\langle R_{a b c d}\right\rangle= & \int d E^{\prime \prime \prime} B_{2}\left(E^{\prime \prime \prime}, T\right) \frac{\hbar}{\Gamma_{a b}} \frac{\hbar k^{\prime \prime \prime}}{\mu_{a, b}} \sigma_{a b}\left(E^{\prime \prime \prime}\right) \\
& \times \int d E^{\prime \prime} B_{2}\left(E^{\prime \prime}, T\right) \frac{\hbar}{\Gamma_{a b, c}} \frac{\hbar k^{\prime \prime}}{\mu_{a b, c}} \sigma_{a b, c}\left(E^{\prime \prime}, E^{\prime \prime \prime}\right) \\
& \times\left\langle R_{a b c, d}\left(E^{\prime \prime}\right)\right\rangle,
\end{aligned}
$$

where $k^{\prime \prime \prime}=\sqrt{2 \mu_{a, b} E^{\prime \prime \prime}} / \hbar$ and $k^{\prime \prime}=\sqrt{2 \mu_{a b, c} E^{\prime \prime}} / \hbar$. Using now Eqs. (5) and (19) we then get

$$
\begin{aligned}
\left\langle R_{a b c d}\right\rangle= & \left(\frac{8}{\pi}\right)^{3 / 2} \frac{\hbar^{2}}{\left(\mu_{a, b} \mu_{a b, c} \mu_{a b c, d}\right)^{1 / 2}} \frac{1}{(K T)^{9 / 2}} \\
& \times \int d E^{\prime \prime \prime} E^{\prime \prime \prime} \frac{\sigma_{a b}\left(E^{\prime \prime \prime}\right)}{\Gamma_{a b}} e^{-\frac{E^{\prime \prime \prime}}{K T}} \\
& \times \int d E^{\prime \prime} E^{\prime \prime} \frac{\sigma_{a b, c}\left(E^{\prime \prime}, E^{\prime \prime \prime}\right)}{\Gamma_{a b, c}} e^{-\frac{E^{\prime \prime}}{K T}} \\
& \times \int d E^{\prime} E^{\prime} \sigma_{a b c, d}\left(E^{\prime}, E^{\prime \prime}, E^{\prime \prime \prime}\right) e^{-\frac{E^{\prime}}{K T}}
\end{aligned}
$$

Finally, using again Eq. (10), we get the following expression for the reaction rate of sequential capture in terms of the inelastic cross section $\sigma_{A d}$ :

$$
\begin{aligned}
\left\langle R_{a b c d}\right\rangle= & \frac{g_{A}}{g_{a b c}}\left(\frac{8}{\pi}\right)^{3 / 2} \frac{\hbar^{2} \mu_{A d}}{\left(\mu_{a, b} \mu_{a b, c}\right)^{1 / 2} \mu_{a b c, d}^{3 / 2}} \frac{1}{(K T)^{9 / 2}} \\
& \times \int d E^{\prime \prime \prime} E^{\prime \prime \prime} \frac{\sigma_{a b}\left(E^{\prime \prime \prime}\right)}{\Gamma_{a b}} e^{-\frac{E^{\prime \prime \prime}}{K T}} \\
& \times \int d E^{\prime \prime} E^{\prime \prime} \frac{\sigma_{a b, c}\left(E^{\prime \prime}, E^{\prime \prime \prime}\right)}{\Gamma_{a b, c}} e^{-\frac{E^{\prime \prime}}{K T}} \\
& \times \int d E^{\prime} T_{A d}^{\prime} \sigma_{A d}\left(T_{A d}^{\prime}\right) e^{-\frac{E^{\prime}}{K T}} .
\end{aligned}
$$

As before, an additional factor $\prod_{i} v_{i}$ ! should be included to account for the presence of identical particles.

\section{B. Two-body resonant cross sections}

The cross sections, $\sigma_{a b}\left(E^{\prime \prime \prime}\right)$ and $\sigma_{a b, c}\left(E^{\prime \prime}, E^{\prime \prime \prime}\right)$, entering in Eq. (22) are just two-body cross sections, which for the case of resonance population can be written as

$$
\sigma_{a b}\left(E^{\prime \prime \prime}\right)=\frac{g_{a b}}{g_{a} g_{b}} \frac{\pi}{k^{\prime \prime \prime 2}} \frac{\Gamma_{a b}^{2}}{\left(E^{\prime \prime \prime}-E_{a b}\right)^{2}+\Gamma_{a b}^{2} / 4}
$$

and

$$
\sigma_{a b, c}\left(E^{\prime \prime}, E^{\prime \prime \prime}\right)=\frac{g_{a b c}}{g_{a b} g_{c}} \frac{\pi}{k^{\prime \prime 2}} \frac{\Gamma_{a b, c}^{2}}{\left(E^{\prime \prime}+E^{\prime \prime \prime}-E_{a b c}\right)^{2}+\Gamma_{a b, c}^{2} / 4},
$$

respectively, where $E_{a b}$ and $E_{a b c}$ are the two- and three-body resonance energies, whose widths are denoted by $\Gamma_{a b}$ and $\Gamma_{a b, c}$, respectively.

Again, the widths are in general energy dependent, a dependence contained in the penetration factor, as indicated in Eq. (13). Also, Eq. (14) is still valid, and we can write down the energy dependence of the widths as

$$
\Gamma_{a b}\left(E^{\prime \prime \prime}\right)=\Gamma_{a b}^{(0)} \frac{1+e^{2 b_{a b} / \sqrt{E_{a b}}}}{1+e^{2 b_{a b} / \sqrt{E^{\prime \prime \prime}}}}
$$

and

$$
\Gamma_{a b, c}\left(E^{\prime \prime}\right)=\Gamma_{a b, c}^{(0)} \frac{1+e^{2 b_{a b, c} / \sqrt{E_{a b c}-E_{a b}}}}{1+e^{2 b_{a b, c} / \sqrt{E^{\prime \prime}}}},
$$

where, for the case of a sequential mechanism, $b_{a b}$ and $b_{a b, c}$ are given by Eqs. (10) and (11) of [15]:

$$
\begin{gathered}
b_{a b, c}=\frac{\pi}{2}\left(Z_{a}+Z_{b}\right) Z_{c} e^{2} \sqrt{\frac{2 \mu_{a b, c}}{\hbar^{2}}}, \\
b_{a b}=\frac{\pi}{2} Z_{a} Z_{b} e^{2} \sqrt{\frac{2 \mu_{a b}}{\hbar^{2}}},
\end{gathered}
$$

and where $Z_{a}, Z_{b}$, and $Z_{c}$ are the charges of particles $a, b$, and $c$, respectively, and $e$ is the electron charge.

If particle $c$ is not charged, the penetration factor takes the form given by Eq. (20) of [15], which gives

$$
\Gamma_{a b, c}\left(E^{\prime \prime}\right)=\Gamma_{a b, c}^{(0)}\left(\frac{E^{\prime \prime}}{E_{a b c}-E_{a b}}\right)^{\ell_{a b, c}+1 / 2},
$$

where $\ell_{a b, c}$ is the relative orbital angular momentum between particle $c$ and the center of mass of the (ab) system.

As in the direct case, we consider also the extreme situation where the width of the $(a b)$ resonance is taken equal to 0 . This implies that

$$
\frac{\sigma_{a b}\left(E^{\prime \prime \prime}\right)}{\Gamma_{a b}} \stackrel{\Gamma_{a b} \rightarrow 0}{\longrightarrow} \frac{2 \pi^{2}}{k_{a b}^{2}} \frac{g_{a b}}{g_{a} g_{b}} \delta\left(E^{\prime \prime \prime}-E_{a b}\right),
$$

where $k_{a b}^{2}=2 \mu_{a, b} E_{a b} / \hbar^{2}$, and which leads to the following expression for the zero-width limit of the reaction rate:

$$
\begin{aligned}
\left\langle R_{a b c d}\right\rangle= & \frac{g_{A}}{g_{a b c}} \frac{g_{a b}}{g_{a} g_{b}}\left(\frac{8}{\pi}\right)^{3 / 2} \frac{\hbar^{4} \pi^{2} \mu_{A d}}{\mu_{a, b}^{3 / 2} \mu_{a b, c}^{1 / 2} \mu_{a b c, d}^{3 / 2}} \frac{e^{-\frac{E_{a b}}{K T}}}{(K T)^{9 / 2}} \\
& \times \int d E^{\prime \prime} E^{\prime \prime} \frac{\sigma_{a b, c}\left(E^{\prime \prime}, E^{\prime \prime \prime}\right)}{\Gamma_{a b, c}} e^{-\frac{E^{\prime \prime}}{K T}} \\
& \times \int d E^{\prime} T_{A d}^{\prime} \sigma_{A d}\left(T_{A d}^{\prime}\right) e^{-\frac{E^{\prime}}{K T}} .
\end{aligned}
$$


If, on top of zero width for the $(a b)$ resonance, we also assume zero width for the $(a b c)$ resonance, we can again make use of Eq. (30), but applied to the $(a b, c)$ system:

$$
\frac{\sigma_{a b, c}\left(E^{\prime \prime}\right)}{\Gamma_{a b, c}} \stackrel{\Gamma_{a b} \rightarrow 0}{\longrightarrow} \frac{2 \pi^{2}}{k_{a b, c}^{2}} \frac{g_{a b c}}{g_{a b} g_{c}} \delta\left(E^{\prime \prime}-\left(E_{a b c}-E_{a b}\right)\right),
$$

where $k_{a b, c}^{2}=2 \mu_{a, b}\left(E_{a b c}-E_{a b}\right) / \hbar^{2}$. After insertion in Eq. (31) we recover the result in Eq. (17), obtained as the extreme limit of the direct capture case.

\section{THEORY REMARKS}

To make easier a general view of the derivations made so far, we consider it useful to collect the key equations used in the actual calculations for both direct and sequential capture mechanisms. We also discuss in this section how the production rates are computed and how they compare with those applying to a radiative capture process.

\section{A. Key equations}

For the direct capture mechanism we summarize the ingredients:

(i) The general expression for the four-body recombination rate is given by Eq. (11).

(ii) The extreme case of zero width for the three-body resonance is given by Eq. (17).

(iii) The cross sections contained in the expressions for the reaction rate are given by Eqs. (2) or (3), depending on the charges of $A$ and $d$, and Eq. (12).

(iv) The width entering in Eq. (12) is given by Eq. (14).

For the sequential capture mechanism we summarize the ingredients:

(i) The general expression for the four-body recombination rate is given by Eq. (22).

(ii) The extreme case of zero width for the two-body resonance, $(a b)$, is given by Eq. (31).

(iii) Zero width for both the $(a b)$ and the $(a b, c)$ systems gives the same limit as in the direct case, Eq. (17).

(iv) The cross sections contained in the expressions for the reaction rate are given by Eqs. (2) or (3), depending on the charges of $A$ and $d$, Eqs. (23) and (24).

(v) The widths entering in Eqs. (23) and (24) are given by Eqs. (25) and (26), respectively.

(vi) If $c$ is not charged, the width in Eq. (24) is given by Eq. (29).

\section{B. Production rates and radiative capture}

When comparing the rate corresponding to different reactions, for instance the production of some nucleus via four-body recombination or three-body radiative capture, this cannot in general be done by simple comparison of the reaction rates. In fact, for a four-body and a three-body process the corresponding reaction rates have different units, making a direct comparison meaningless. The comparison has to be done in terms of the production rates, which give the number of reaction products created per unit time and unit volume.
The production rates are obtained from the reaction rates after multiplication by the number density of each of the particles involved in the reaction. This density is given by $n_{i}=\rho N_{A} X_{i} / A_{i}$, where $\rho$ is the mass density, $N_{A}$ is the Avogadro number, $X_{i}$ is the mass abundance of particle $i$, and $A_{i}$ is the atomic number of particle $i$.

The ratio between the production rates corresponding to the four-body recombination, $P_{\mathrm{rec}}$, and the one corresponding to a radiative capture process, $P_{\text {rad }}$, leading both to the same final product, can be then easily computed. In fact, it is obvious that this ratio, $P_{\text {rec }} / P_{\text {rad }}$, grows linearly with the number density of the spectator particle in the four-body reaction. In other words, it grows linearly with the mass density in the environment where the reactions take place. Therefore, it is clear that for a sufficiently high value of the density, the four-body recombination process will dominate over the radiative capture.

In Ref. [5] an analysis similar to the one shown in Secs. III and IV was made for the radiative capture process $a+b+c \rightarrow A+\gamma$. Analogous expressions were obtained for the cases of direct and sequential capture mechanism (Eqs. (3) and (10) in Ref. [5]). In particular, it is worth noticing that Eq. (15) of Ref. [5] gives a simple analytical expression for the radiative capture process in the limit of zero width for the two- and three-body resonances. This expression is therefore the equivalent to Eq. (17) for the four-body recombination reaction in the same limit. Therefore, use of the reaction rates in Eq. (17) and in Eq. (15) of Ref. [5] permits us to obtain a simple expression for $P_{\text {rec }} / P_{\text {rad }}$ in the extreme case of making the widths of the resonances equal to zero. This ratio takes the form

$$
\begin{aligned}
\frac{P_{\mathrm{rec}}}{P_{\mathrm{rad}}} \stackrel{\Gamma_{a b c} \rightarrow 0}{\longrightarrow} & n_{d} \frac{g_{A}}{\mu_{A d}^{1 / 2}} \sqrt{\frac{8}{\pi}} \frac{\hbar}{\Gamma_{\gamma}\left(E_{a b c}\right)} \frac{1}{(K T)^{3 / 2}} \\
& \times \int_{0}^{\infty} d E^{\prime} T_{A d}^{\prime} \sigma_{A d}\left(T_{A d}^{\prime}\right) e^{-\frac{E^{\prime}}{K T}}
\end{aligned}
$$

where $n_{d}$ is the number density of the spectator particle $d$, $\tau_{\gamma}=\hbar / \Gamma_{\gamma}\left(E_{a b c}\right)$ is the lifetime for $\gamma$ decay of the $(a b c)$ resonance, and $T_{A d}^{\prime}=E^{\prime}-\left(B-E_{a b c}\right)$.

The expression (33) is the one actually used in Refs. [6-8,11] for the production rate ratio between the particle induced de-excitation process and the radiative capture process. It is then clear that in those works the effect of the resonance widths and the reaction mechanism are neglected, in such a way that the only key ingredient entering is the $\sigma_{A d}$ cross section. We can then conclude that, provided the same $\sigma_{A d}$ cross sections are used, the results in Refs. [6-8,11] will be the same as obtained in this work in the limit of zero resonance widths. As we will see, the effect of the resonance widths will be visible only in the low temperature region, which implies that for high temperatures, where Eq. (33) is a good approximation, no relevant differences between our results and the ones in Refs. [6-8,11] are expected.

Finally, let us close this section mentioning that in Eq. (33) it is assumed that the four-body and the three-body processes involve the same number of identical particles (for instance the $\alpha+\alpha+\alpha+n \rightarrow{ }^{12} \mathrm{C}+n$ and $\alpha+\alpha+\alpha \rightarrow$ ${ }^{12} \mathrm{C}+\gamma$ processes). However, other situations are possible, 
like having four identical particles in the four-body recombination process (for instance the $\alpha+\alpha+\alpha+\alpha \rightarrow{ }^{12} \mathrm{C}+\alpha$ process), in which case the ratio in Eq. (33) should be multiplied by 4 . In general, Eq. (33) has to be multiplied by the factor $\prod_{i} v_{i}$ !, corresponding to the four-body particle induced de-excitation process, and divided by the same factor, $\prod_{i} v_{i}$ !, corresponding to the three-body radiative process. As before, for each of the two reactions, $v_{i}$ is the number of identical particles of type $i$.

\section{THE CASES OF ${ }^{9}$ Be AND ${ }^{12} \mathrm{C}$}

In this section we apply the results previously described to four-body recombination processes leading to the formation of ${ }^{9} \mathrm{Be}(\alpha+\alpha+n)$ and ${ }^{12} \mathrm{C}(\alpha+\alpha+\alpha)$. The cases where a neutron, a proton, or an $\alpha$ particle act as spectator will be considered.

\section{A. Cross sections}

The crucial ingredients for the calculation of the reaction rates are the cross sections contained in the expressions (11) and (22) corresponding to the direct and sequential mechanisms, respectively.

As mentioned, two different types of cross sections appear. The first type corresponds to population of a two- or a three-body resonance, which are given by Eq. (12) for direct capture, and Eqs. (23) and (24) for sequential capture. These cross sections are fully specified by the corresponding resonance energies and widths.

For the two cases considered in this work, ${ }^{9} \mathrm{Be}$ and ${ }^{12} \mathrm{C}$, we shall include in the calculation the contribution from the two three-body resonances contributing the most to reaction rates. As in the case of radiative capture [5], the effect of the second resonance is expected to play a role only for high temperatures. In this temperature region there could also be some small effects from even higher resonances, which could interfere through the resonance tails. This effect is not very relevant for the purpose of this work, and therefore it will not be considered here.

In the case of ${ }^{9} \mathrm{Be}$, we shall consider the contributions to the rate from the $1 / 2^{+}$and $5 / 2^{+}$three-body resonances decaying into the $3 / 2^{-}$ground state of ${ }^{9} \mathrm{Be}$. The contribution from the $1 / 2^{+}$state is expected to clearly dominate, although, as shown in Ref. [10], the contribution from the $5 / 2^{+}$resonance could be important at high temperatures. The energies and widths $\left(E_{\text {res }}, \Gamma_{\text {res }}\right)$ employed for the $1 / 2^{+}$and $5 / 2^{+}$resonances are, respectively, $(0.110,0.217) \mathrm{MeV}$ and $(1.475,0.282) \mathrm{MeV}$ [16]. The binding energy of the ${ }^{9} \mathrm{Be}$ ground state is $B=$ $-1.574 \mathrm{MeV}$ [16].

In case of sequential capture, the process is assumed to take place through the $0^{+}$resonance in ${ }^{8} \mathrm{Be}$, whose energy and width are, respectively, $0.092 \mathrm{MeV}$ and $5.6 \times 10^{-6} \mathrm{MeV}$ [16]. In principle, the sequential formation of ${ }^{9} \mathrm{Be}$ could also take place through the lowest, $\frac{3}{2}^{-}$, resonance in ${ }^{5} \mathrm{He}(\alpha+n)$. However, this resonance is located at $0.798 \mathrm{MeV}$ above threshold [17], being then much less energetically accessible than the significantly lower $0^{+}$resonance in ${ }^{8} \mathrm{Be}$. In fact, even for temperatures of $1 \mathrm{GK}$ the Maxwell-Boltzmann distribution peaks
TABLE I. Resonance energies, $E_{\text {res }}$, and widths, $\Gamma_{\text {res }}$, in $\mathrm{MeV}$, for the two- and three-body resonances included in the calculations of the reaction rates in Eq. (11), direct capture, and Eq. (22), sequential capture, for the formation of ${ }^{9} \mathrm{Be}$ and ${ }^{12} \mathrm{C}$.

\begin{tabular}{lccccc}
\hline \hline & ${ }^{8} \mathrm{Be}$ & \multicolumn{2}{c}{${ }^{9} \mathrm{Be}$} & \multicolumn{2}{c}{${ }^{12} \mathrm{C}$} \\
\hline$J^{\pi}$ & $0^{+}$ & $1 / 2^{+}$ & $5 / 2^{+}$ & $0^{+}$ & $2^{+}$ \\
$E_{\text {res }}$ & 0.092 & 0.110 & 1.475 & 0.38 & 1.84 \\
$\Gamma_{\text {res }}$ & $5.6 \times 10^{-6}$ & 0.217 & 0.282 & $9.3 \times 10^{-6}$ & 0.56 \\
\hline \hline
\end{tabular}

at an energy even below $0.5 \mathrm{MeV}$. Furthermore, the large width of the $\frac{3}{2}^{-}$resonance in ${ }^{5} \mathrm{He}(0.648 \mathrm{MeV}$, [17]) makes the capture of the second $\alpha$ particle before the resonance decay very unlikely, and, at the same time, smears out the resonance contribution to the reaction rate into the continuum background. For these reasons the sequential capture through ${ }^{5} \mathrm{He}$ intermediate states will not be considered here.

For ${ }^{12} \mathrm{C}$, the contributions to the rate from the $0^{+}$and $2^{+}$three-body resonances decaying into the $0^{+}$ground state will be considered. The energy and width employed for the Hoyle $0^{+}$state are $\left(0.38,9.3 \times 10^{-6}\right) \mathrm{MeV}$ [18]. For the lowest $2^{+}$resonance we take $(1.84,0.56) \mathrm{MeV}$, as suggested in Ref. [2]. The required binding energy of the ${ }^{12} \mathrm{C}$ ground state is $B=-7.275 \mathrm{MeV}$ [18]. In case of sequential capture, the process is again taking place through the $0^{+}$resonance in ${ }^{8} \mathrm{Be}$. The energies and widths of the resonances included in the calculation are collected in Table I.

The second type of cross sections are the inelastic $\sigma_{A d}$ cross sections. They will be assumed to take the form given in Eqs. (2) and (3) for the cases where particle $d$ is charged or neutral, respectively. Furthermore, we know that the cross section must be equal to zero for collision energies smaller than $|B|$, where $B$ is the binding energy of the $A$ system. To guarantee this condition, we multiply the cross sections (2) and (3) by the shape function:

$$
f(E)=\frac{e^{(E-|B|) / a}-1}{e^{(E-|B|) / a}+1},
$$

which is 0 for $E=|B|$ and 1 for $E \gg|B|$. The diffuseness parameter $a$ determines how fast the transition from 0 to 1 takes place.

The key point here is how to determine the $S$ factors, which should be estimated for the cases of incident proton, neutron, or $\alpha$ particle on both, ${ }^{9} \mathrm{Be}$ and ${ }^{12} \mathrm{C}$. In this work we shall take $S(E)=S_{0}(1+\gamma E)$, where $S_{0}$ and $\gamma$ are constants to be extracted from the available experimental information.

In Ref. [2], the $S$ factor for quite a few reactions are plotted. Among them the one for the reaction ${ }^{9} \mathrm{Be}(p, n){ }^{9} \mathrm{~B}$. The $S$ factor corresponding to the proton- ${ }^{9} \mathrm{Be}$ collision grows fast from zero and it soon reaches a rather constant value of about $10 \mathrm{MeV} \times$ barn. This value seems not to depend much on the charge of the target nucleus, as seen for instance for the ${ }^{11} \mathrm{~B}(p, n){ }^{11} \mathrm{C}$ and ${ }^{13} \mathrm{C}(p, n){ }^{13} \mathrm{~N}$ reactions, where a similar constant value of the $S$ factor is seen. Therefore, for the case of incident proton we take $S_{0}=10 \mathrm{MeV} \times$ barn for both, ${ }^{9} \mathrm{Be}$ and ${ }^{12} \mathrm{C}$. This value of $S_{0}$ together with a diffuseness parameter $a=0.9 \mathrm{fm}$ in Eq. (34), are enough to reproduce reasonably 

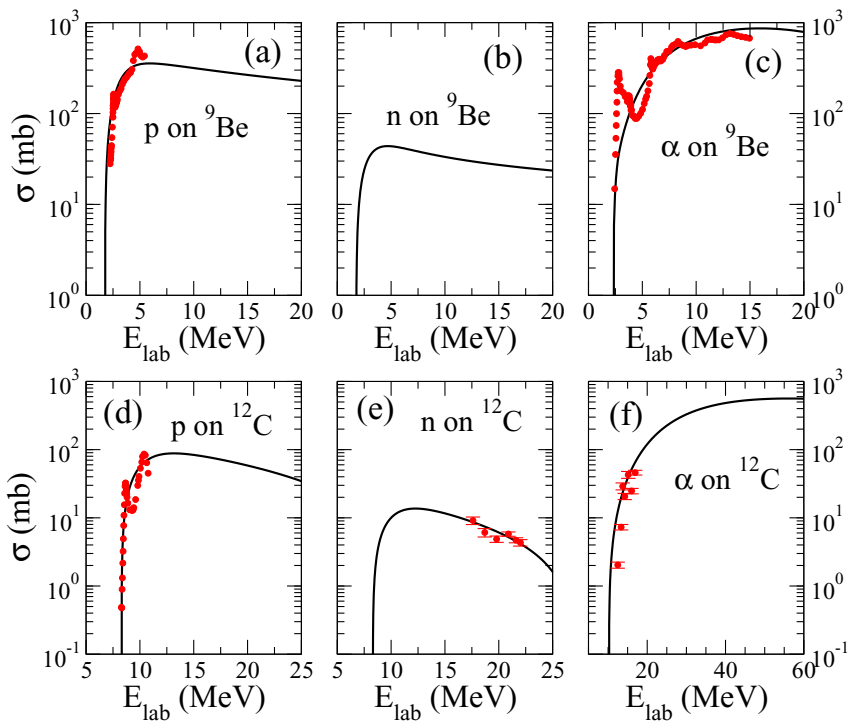

FIG. 1. Cross sections, in mb, as a function of the laboratory energy of the incident particle, for proton, neutron, and $\alpha$ on ${ }^{9} \mathrm{Be}$, (a)-(c), respectively, and for proton, neutron, and $\alpha$ on ${ }^{12} \mathrm{C}$, (d)-(f), respectively. The experimental data (solid circles) are from [19] and [20] in (a) and (c), and from Refs. [8], [21], and [7], in (d)-(f), respectively.

well the experimental values of the cross section given in Ref. [19] for the case of ${ }^{9} \mathrm{Be}$ target. The computed cross section (solid line) and the experimental data (solid circles) are shown in Fig. 1(a). For the case of ${ }^{12} \mathrm{C}$, the comparison with the experimental data in Ref. [8], Fig. 1(d), suggests a bigger value of the diffuseness parameter, $a=1.5 \mathrm{fm}$, and a small correction of the $S$ factor by taking $\gamma=-0.032 \mathrm{MeV}^{-1}$. The introduction of $\gamma$ gives rise to a fall off of the cross section for large energies similar to the one shown in Ref. [11].

For the case of incident neutron the value of $S_{0}$ is given in Ref. [12] for several different reactions. However, its value oscillates quite a lot from one reaction to another. For instance for the reactions ${ }^{7} \mathrm{Be}(n, p){ }^{7} \mathrm{Li}$ and ${ }^{14} \mathrm{~N}(n, p){ }^{14} \mathrm{C}$ the difference in the given $S_{0}$ is of almost five orders of magnitude. As a consequence, it is not easy to extract a more or less reliable value to be used in our calculations. Therefore, for each of the two cases, ${ }^{9} \mathrm{Be}$ and ${ }^{12} \mathrm{C}$, we have opted for using the same diffuseness parameter $a$ as in the proton case, and fine tune $S_{0}$ and $\gamma$ such that the experimental values given in Ref. [21] for the case of ${ }^{12} \mathrm{C}$ are reasonably well reproduced. This is done by taking $S_{0}=0.1 \mathrm{MeV}^{1 / 2} \times$ barn and $\gamma=-0.04 \mathrm{MeV}^{-1}$, as shown in Fig. 1(e). Thus, for the case of ${ }^{9} \mathrm{Be}$ we have also used $S_{0}=0.1 \mathrm{MeV}^{1 / 2} \times$ barn, whereas $\gamma$ is taken equal to zero as in the proton case. For completeness we show in Fig. 1(b) the corresponding cross section.

Finally, in Ref. [2] the $S$ factor for the ${ }^{9} \mathrm{Be}(\alpha, n){ }^{12} \mathrm{C}$ reaction is also shown. In this case the $S$ factor is not as constant as a function of the energy as in the proton case, but for an energy in the vicinity of $1.5 \mathrm{MeV}$ (needed to excite the ${ }^{9} \mathrm{Be}$ nucleus), it takes a value that oscillates around $10^{3} \mathrm{MeV} \times$ barn. A similar value is observed for the ${ }^{13} \mathrm{C}(\alpha, n){ }^{16} \mathrm{O}$ and the ${ }^{14} \mathrm{~N}(\alpha, n){ }^{17} \mathrm{~F}$ reactions. Therefore, for the alpha particle we
TABLE II. Parameters used for the inelastic cross sections $\sigma_{A d}$ for the cases of incident proton, neutron, and $\alpha$ on ${ }^{9} \mathrm{Be}$ and ${ }^{12} \mathrm{C} . S_{0}$ is given in units of $\mathrm{MeV} \times$ barn for incident protons and $\alpha \mathrm{s}$, and in units of $\mathrm{MeV}^{1 / 2} \times$ barn for the case of incident neutron. The parameters $\gamma$ and $a$ are given in $\mathrm{MeV}^{-1}$ and fm, respectively.

\begin{tabular}{ccccccccc}
\hline \hline & \multicolumn{2}{c}{ proton } & & \multicolumn{2}{c}{ neutron } & & \multicolumn{2}{c}{ alpha } \\
\cline { 2 - 3 } \cline { 8 - 9 } \cline { 8 - 9 } & ${ }^{9} \mathrm{Be}$ & ${ }^{12} \mathrm{C}$ & & ${ }^{9} \mathrm{Be}$ & ${ }^{12} \mathrm{C}$ & & ${ }^{9} \mathrm{Be}$ & ${ }^{12} \mathrm{C}$ \\
\hline$S_{0}$ & 10 & 10 & & 0.1 & 0.1 & & $10^{3}$ & $10^{3}$ \\
$\gamma$ & 0 & -0.032 & & 0 & -0.040 & & -0.045 & -0.010 \\
$a$ & 0.9 & 1.5 & & 0.9 & 1.5 & & 0.1 & 8.0 \\
\hline \hline
\end{tabular}

take $S_{0}=10^{3} \mathrm{MeV} \times$ barn for both ${ }^{9} \mathrm{Be}$ and ${ }^{12} \mathrm{C}$. The main difference between these two cases is that in order to reproduce the experimental cross sections (Ref. [20] for ${ }^{9} \mathrm{Be}$ and Ref. [7] for ${ }^{12} \mathrm{C}$ ) a quite small value of $a$ is needed for ${ }^{9} \mathrm{Be}$ $(a=0.1 \mathrm{fm})$ producing a very sharp increase of the cross section at small energies. However, for ${ }^{12} \mathrm{C}$ the increase of the cross section is much smoother, and actually a rather large value of $a$ is needed ( $a=8 \mathrm{fm}$ ). These values of the diffuseness parameter, together with $\gamma=-0.045 \mathrm{MeV}^{-1}$ for ${ }^{9} \mathrm{Be}$ and $\gamma=-0.010 \mathrm{MeV}^{-1}$ for ${ }^{12} \mathrm{C}$, give rise to the cross sections shown by the solid curves in Figs. 1(c) and 1(f), respectively, where the experimental data are given by the solid circles. All the parameters used for the different inelastic cross sections discussed above are collected in Table II.

It is important to note that the experimental data shown in Fig. 1 obviously correspond to collisions of the $\alpha$, proton, or neutron projectile on the target in its ground state. The uncertainties introduced by using the same cross sections for the case of collisions on an excited state of the target (like for instance the bound excited $2^{+}$state in ${ }^{12} \mathrm{C}$ ) are big, and could lead to very unrealistic results. For this reason we have considered here decays of the three- $\alpha$ resonances into the ground state of ${ }^{12} \mathrm{C}$ only.

\section{B. Results for ${ }^{9} \mathrm{Be}$}

Let us now make use of the cross sections described in the previous section and consider the four-body recombination processes $\alpha+\alpha+n+\alpha \rightarrow{ }^{9} \mathrm{Be}+\alpha, \alpha+\alpha+n+n \rightarrow$ ${ }^{9} \mathrm{Be}+n$, and $\alpha+\alpha+n+p \rightarrow{ }^{9} \mathrm{Be}+p$, where either an $\alpha$ particle, or a neutron, or a proton, respectively, act as spectators inducing the formation of ${ }^{9} \mathrm{Be}$.

In Fig. 2 the solid curves give, for each of the three cases, panels (a), (b), and (c), respectively, the four-body recombination rate obtained assuming a direct capture mechanism, as considered when deriving Eq. (11). In the same way, the dashed curves show the same rates but assuming a sequential mechanism, i.e., using Eq. (22). Whereas the thick curves contain the contribution form both, the $1 / 2^{+}$and the $5 / 2^{+}$ resonances in ${ }^{9} \mathrm{Be}$, the thin curves contain the contribution from the $1 / 2^{+}$resonance only.

As we can see, in all the three cases, $\alpha$, neutron, and proton spectator, the direct mechanism dominates for very small energies, but for values of $K T$ larger than about $0.1 \mathrm{MeV}$ $(T \approx 1 G K)$ the sequential mechanism always dominates. We 


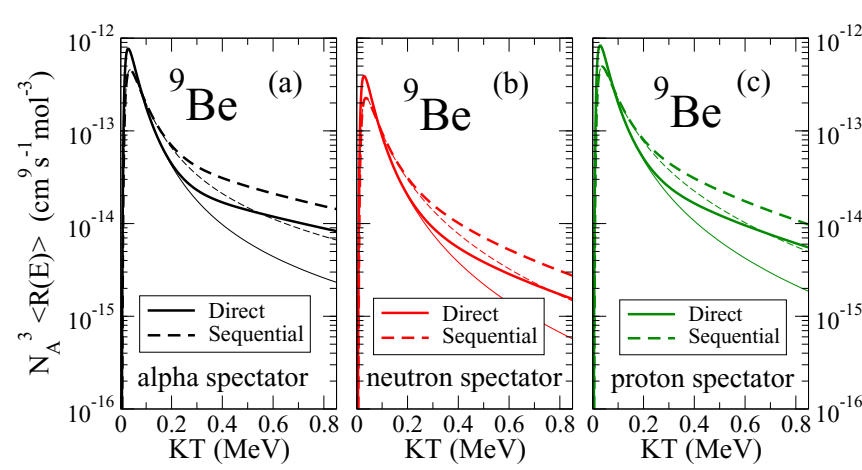

FIG. 2. Four-body recombination rates for (a) the $\alpha+\alpha+n+$ $\alpha \rightarrow{ }^{9} \mathrm{Be}+\alpha$, (b) the $\alpha+\alpha+n+n \rightarrow{ }^{9} \mathrm{Be}+n$, and (c) the $\alpha+$ $\alpha+n+p \rightarrow{ }^{9} \mathrm{Be}+p$ reactions as a function of $K T$ (in $\mathrm{MeV}$ ). The solid and dashed curves show the cases of direct and sequential capture, respectively. The corresponding thin curves give the reaction rate including the contribution from the $1 / 2^{+}$resonance only. $N_{A}$ is the Avogadro's number.

also observe that the cases of alpha and proton as spectator produce similar reaction rates, which dominate over the case of having a neutron as spectator particle. Finally, we also see that the effect of the $5 / 2^{+}$resonance contribution is clearly visible in all the cases and capture mechanisms for $K T$ values beyond about $0.2 \mathrm{MeV}$.

The format in Fig. 2 has been chosen to make easier the comparison with the right part of Fig. 3 in Ref. [10]. As we can see the result obtained here is consistent with the one shown in Ref. [10] for the $1 / 2^{+}$resonance, which is by far the dominating contribution. This is like this in both cases, when the $\alpha$ particle is the spectator (thin-dashed curve in Ref. [10]), and when the neutron is the spectator (thick-dashed curve in Ref. [10]). The case of proton spectator was not considered in Ref. [10].

In order to make easier the comparison between the different curves at very low temperatures we show in Fig. 3 the

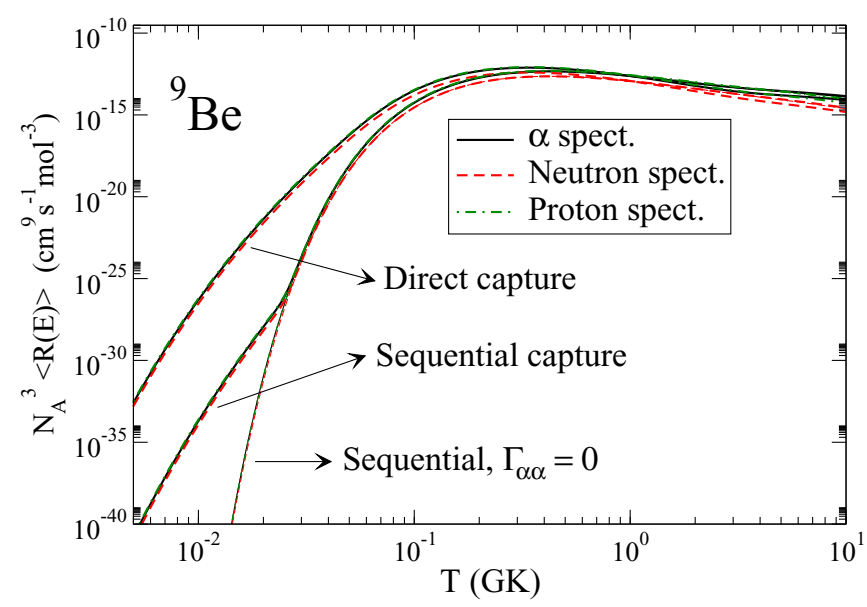

FIG. 3. Same reaction rates as in Fig. 2 as a function of $T$ and logarithmic scale. The solid, dashed, and dot-dashed curves show the results obtained with an alpha, a neutron, or a proton as spectator particle. The cases of direct, sequential, and extreme sequential $\left(\Gamma_{\alpha \alpha}=0\right.$, Eq.(31)) capture are shown. $N_{A}$ is the Avogadro's number.

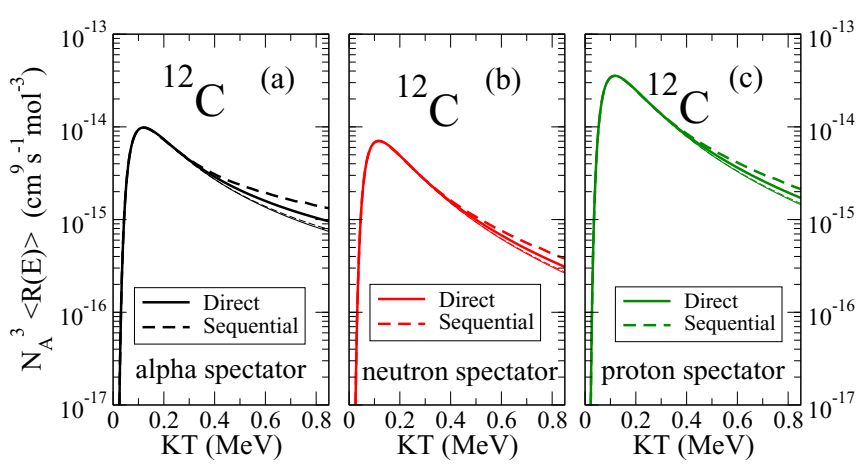

FIG. 4. Four-body recombination rates for (a) the $\alpha+\alpha+\alpha+$ $\alpha \rightarrow{ }^{12} \mathrm{C}+\alpha$, (b) the $\alpha+\alpha+\alpha+n \rightarrow{ }^{12} \mathrm{C}+n$, and (c) the $\alpha+$ $\alpha+\alpha+p \rightarrow{ }^{12} \mathrm{C}+p$ reactions as a function of $K T$ (in $\mathrm{MeV}$ ). The solid and dashed curves show the cases of direct and sequential capture, respectively. The corresponding thin curves give the reaction rate including the contribution from the Hoyle $0^{+}$resonance only. $N_{A}$ is the Avogadro's number.

same reaction rates as in Fig. 2 but using a logarithmic scale in the $x$ axis, where the temperature (in $\mathrm{GK}$ ) is given. In the figure we also show the extreme cases where the width of the two-body $(\alpha \alpha)$ resonance is taken equal to zero in the case of a sequential process. We can see that for low temperatures the rate corresponding to a direct mechanism can be up to about 8 orders of magnitude bigger than the one corresponding to the sequential process. Also, a comparison between the extreme sequential $\left(\Gamma_{\alpha \alpha}=0\right)$ and the sequential cases permits us to observe that the effect of the width of the $(\alpha \alpha)$ resonance is visible only for temperatures smaller than $\approx 0.02 \mathrm{GK}$, whereas for higher temperatures the effect of the two-body resonance width is negligible. In any case, the dominance of one capture mechanism versus the other could lead to an important change of the reaction rate at low temperatures of several orders of magnitude, as also found in Ref. [5] for the case of radiative capture.

\section{Results for ${ }^{12} \mathrm{C}$}

Let us now consider the processes leading to the formation of ${ }^{12} \mathrm{C}$, i.e., the reactions $\alpha+\alpha+\alpha+\alpha \rightarrow{ }^{12} \mathrm{C}+\alpha$, $\alpha+\alpha+\alpha+n \rightarrow{ }^{12} \mathrm{C}+n$, and $\alpha+\alpha+\alpha+p \rightarrow{ }^{12} \mathrm{C}+p$

The results are shown in Fig. 4, where again the solid and dashed curves represent, respectively, the reaction rates obtained assuming a direct or a sequential capture mechanism. The thin curves give the result including only the transition from the Hoyle $0^{+}$resonance. From the figure we can emphasize the following results. First, we see now a much smaller variation compared to the case of ${ }^{9} \mathrm{Be}$ between the direct and sequential pictures, although still the sequential mechanism tends to dominate at high temperatures. Second, the peak of the reaction rates is even more than an order of magnitude smaller than for ${ }^{9} \mathrm{Be}$. Third, the case of having a proton as spectator produces now a clearly bigger rate than when the spectator is either a neutron or an alpha particle. For the case of ${ }^{9} \mathrm{Be}$ the rates obtained for the proton and $\alpha$ spectators were very similar to each other. And finally, fourth, similarly to what happens with the $5 / 2^{+}$resonance in ${ }^{9} \mathrm{Be}$, for ${ }^{12} \mathrm{C}$ the 


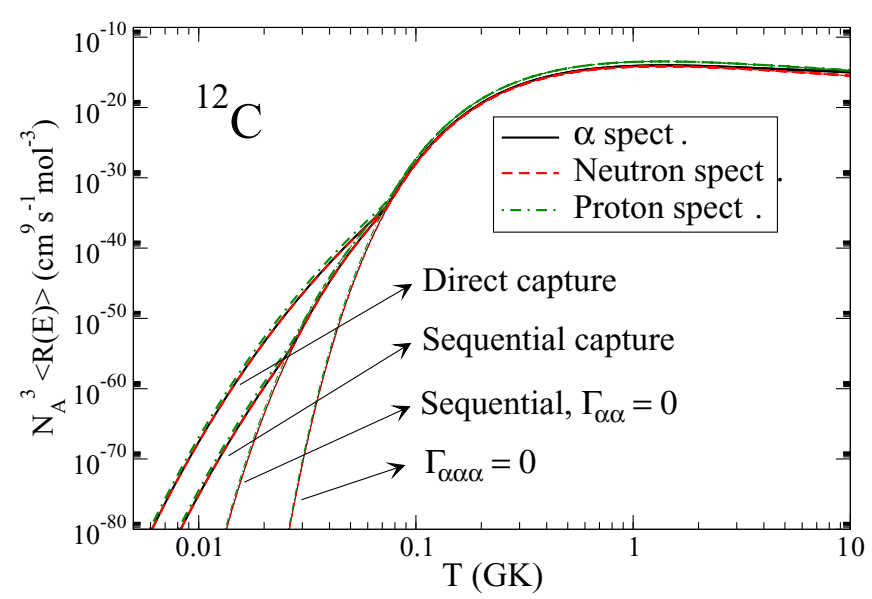

FIG. 5. Same reaction rates as in Fig. 4 as a function of $T$ and logarithmic scale. The solid, dashed, and dot-dashed curves show the results obtained with an $\alpha$, a neutron, or a proton as spectator particle. Together with the cases of direct, sequential, and extreme sequential $\left[\Gamma_{\alpha \alpha}=0\right.$, Eq. (31)] capture mechanisms, we show here as well the extreme case of zero width for the three-body resonance $\left[\Gamma_{\alpha \alpha \alpha}=0\right.$, Eq. (17)]. $N_{A}$ is the Avogadro's number.

contribution of the $2^{+}$resonance shows up for values of $K T$ beyond $0.4 \mathrm{MeV}$, although in this case the increase produced in the reaction rate is more modest.

We again emphasize the differences between the different scenarios at low temperatures by introducing a logarithmic scale in the temperature axis. This is shown in Fig. 5, where the meaning of the different curves is as in Fig. 3, although in this case we have included as well the limit in Eq. (17), obtained assuming the width of the three-body resonance (the Hoyle state in our case) equal to zero. As we can see, at high temperatures (above $0.1 \mathrm{GK}$ ) the distinction between the results obtained with the sequential and direct mechanisms is hardly seen. Below this temperature we observe an effect of the three-body resonance width, as indicated by the jump in the reaction rates corresponding to the $\Gamma_{\alpha \alpha \alpha}=0$ limit and the other scenarios. For temperatures below about $0.02 \mathrm{GK}$ the effect of the two-body resonance width is seen in the sequential case (jump from the $\Gamma_{\alpha \alpha}=0$ curves and the ones corresponding to the full sequential capture mechanism).

All in all, again a dominance of the direct capture mechanism can produce a significant increase of the reaction rates at very low temperatures, increase that can reach up to seven orders of magnitude compared to the case of dominance of the sequential capture mechanism.

\section{Productions rates}

As discussed in Sec. V B, the production rates are obtained after multiplication of the reaction rates by the density of each of the particles involved in the reaction, i.e., $n_{i}=\rho N_{A} X_{i} / A_{i}$, where $\rho$ is the mass density, $N_{A}$ is the Avogadro number, $X_{i}$ is the mass abundance of particle $i$, and $A_{i}$ is the atomic number of particle $i$. This therefore implies that the ratio between the four-body recombination and the radiative three-body production rates will be proportional to the density $n_{d}$ of the spectator particle $d$ in the four-body process. In other words, it will be
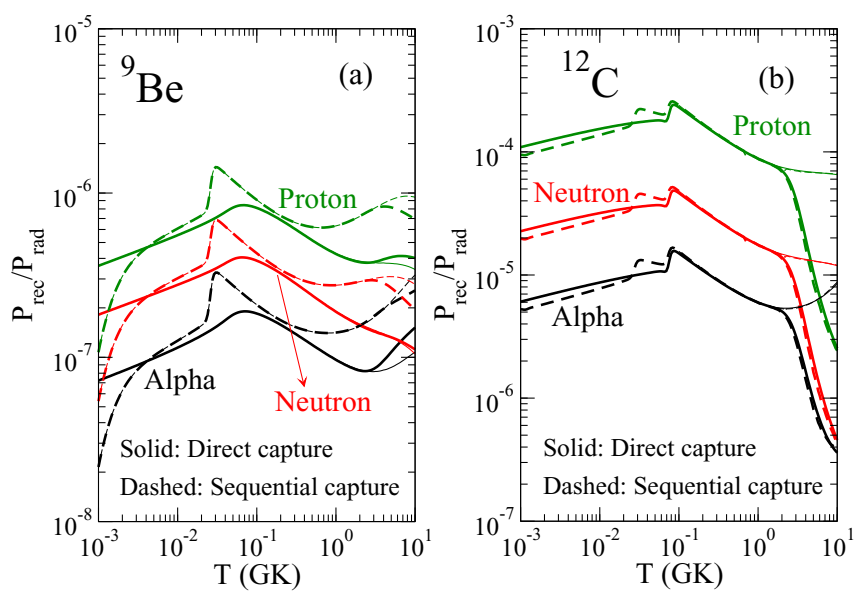

FIG. 6. Ratio between the four-body recombination production rates giving rise to ${ }^{9} \mathrm{Be}$ (a) and ${ }^{12} \mathrm{C}(\mathrm{b})$, and the respective radiative capture processes $\alpha+\alpha+n \rightarrow{ }^{9} \mathrm{Be}+\gamma$ and $\alpha+\alpha+\alpha \rightarrow{ }^{9} \mathrm{Be}+$ $\gamma$. A mass density $\rho=1 \mathrm{~g} / \mathrm{cm}^{3}$ and spectator abundance $X_{d}=1$ are assumed. In both panels, the solid and dashed curves correspond to direct and sequential capture mechanisms, respectively. The cases of an $\alpha$, a neutron, or a proton as spectator in the four body process are shown. The thin curves are obtained excluding the contributions from the $5 / 2^{+}$resonance in the ${ }^{9} \mathrm{Be}$ case, and the $2^{+}$resonance in the ${ }^{12} \mathrm{C}$ case.

proportional to the mass density $\rho$ and the mass abundance $X_{d}$ of the spectator particle.

In what follows we shall take $\rho=1 \mathrm{~g} / \mathrm{cm}^{3}$ and $X_{d}=1$, in such a way that to get the production rate ratio for given values of $\rho$ and $X_{d}$ one should simply multiply the computed curves by $\rho X_{d}$ (with $\rho$ given in $\mathrm{g} / \mathrm{cm}^{3}$ ).

In Fig. 6(a) we show, as a function of the temperature, the ratio between the production rates corresponding to the four-body recombination processes giving rise to ${ }^{9} \mathrm{Be}$, and the production rate corresponding to the radiative capture process $\alpha+\alpha+n \rightarrow{ }^{9} \mathrm{Be}+\gamma$. We take the radiative production rates obtained as described in Ref. [5]. The solid and dashed curves show, respectively, the results assuming a direct or a sequential capture mechanism. The cases of alpha, neutron, or proton as spectator particle in the four-body reaction are shown.

As we can see, for the mass density used in the calculation, $\rho=1 \mathrm{~g} / \mathrm{cm}^{3}$, the particle induced recombination is far of dominating. As anticipated in Ref. [10], densities of about $\rho=10^{6}$ or $10^{7} \mathrm{~g} / \mathrm{cm}^{3}$ are needed for the four-body recombination to dominate (provided that the abundance $X_{d}$ of the spectator particle is close to 1). This is particularly true for high temperatures in case of sequential capture. The kink observed at about $0.02 \mathrm{GK}$ for the sequential mechanism (dashed curves) is a consequence of the kink observed at the same temperature in the reaction rates, as seen in Fig. 3. We also show in the figure the ratio obtained after excluding the contribution from the $5 / 2^{+}$resonance (thin curves). Only a minor effect is seen for temperatures close to $1 \mathrm{GK}$.

In Fig. 6(b) the same ratio is shown but for the reactions leading to the formation of ${ }^{12} \mathrm{C}$. The meaning of the curves is as in Fig. 6(a). Comparing with the ${ }^{9} \mathrm{Be}$ case we observe that in this case the difference between the direct and sequential capture mechanisms is now much smaller. This fact was 
already observed in Fig. 4, where the reaction rates are shown to depend on the reaction mechanism much less that in the ${ }^{9}$ Be case (Fig. 2).

Another relevant feature observed in Fig. 6(b) is that the production rate ratio is now about two orders of magnitude bigger than in panel (a) for all the cases. This implies that densities about two orders of magnitude smaller, $10^{4}$ or $10^{5} \mathrm{~g} / \mathrm{cm}^{3}$, could be sufficient to make the four-body process dominate (again assuming $X_{d} \approx 1$ ). This is especially true at intermediate energies, since for energies approaching $1 \mathrm{GK}$ a rather pronounced drop of the ratio is found. This is due to the fact that for these temperatures the contribution from the $2^{+}$resonance in ${ }^{12} \mathrm{C}$ becomes important, and it has a clearly more significant weight for the radiative capture process than for the particle induced recombination. The consequence is the observed fall in the ratio. This can be better understood by looking at the same ratio but excluding the contribution of the $2^{+}$resonance from the calculation. In this case, thin curves in the figure, the drastic decrease of the ratio disappears. It is then clear that an unambiguous determination of the energy and width of the $2^{+}$resonance in ${ }^{12} \mathrm{C}$ is crucial in order to determine the production rate ratio at high temperatures.

As discussed, our results for high temperatures, where the zero-width limit given by Eq. (33) is a good approximation, should be consistent with the previous results for ${ }^{12} \mathrm{C}$ shown in Refs. [7,8,11], where Eq. (33) is actually used. From Fig. 6(b) we can see that for temperatures around $10 \mathrm{GK}$ and a mass density of $\rho=10^{6} \mathrm{~g} / \mathrm{cm}^{3}$, in the case of neutron or proton as spectator, the production rate for the four-body recombination process is within factors of 10 to 100 times the production rate for the radiative capture process, which is consistent with the value of about 20 to 30 given in Refs. [8,11]. For the case of $\alpha$ spectator and the same mass density, the same factor ranges within 1 to 10 , which can also be considered consistent with the results in Refs. [7,11], where they report values of about 3 and 0.3 , respectively. The differences between our results and the ones in Refs. [7,8,11] can be easily understood as due to the uncertainties in the $\sigma_{A d}$ cross sections (Fig. 1), and the ones arising by the role played by the $2^{+}$resonance, as illustrated by the difference between the thick and thin curves in Fig. 6(b) at high temperatures. At low temperatures, however, where Eq. (33) does not hold anymore, our results and the ones in Refs. [7,8,11] are very different. Whereas for $T=10^{-3} \mathrm{GK}$ we predict production rate ratios that for $\rho=10^{6} \mathrm{~g} / \mathrm{cm}^{3}$ range from 10 to 100 , the corresponding ratio in Refs. $[7,8,11]$ is essentially zero. This is the consequence of taking into account the combined effect of the resonance width and the possibility of a direct capture mechanism.

Finally, let us mention that in Fig. 6(b) we observe one kink in the curves at about $0.08 \mathrm{GK}$ for both, the direct and sequential mechanisms, and an additional one at about 0.02 GK for the sequential case. They reflect the corresponding kinks observed in Fig. 5, at the same temperatures, for each of the capture mechanisms.

\section{SUMMARY AND CONCLUSIONS}

In this work we have derived analytical expressions for the particle induced recombination reaction rates taking into account the two possible capture mechanisms, sequential and direct, with and without population of an intermediate twobody state.

The derived reaction rates contain several cross sections that are described through simple phenomenological expressions. In particular, for those cross sections describing the population of either a two-body or a three-body resonance a Breit-Wigner form is used. For the cross sections describing the inelastic excitation of the final bound product a standard energy-dependent form in terms of the $S$ factor is employed.

We apply the theoretical framework to the case of production of ${ }^{9} \mathrm{Be}$ and ${ }^{12} \mathrm{C}$ in an environment containing neutrons, protons, and $\alpha$ particles. The contribution from the $1 / 2^{+}$and $5 / 2^{+}$resonances are considered for the case of ${ }^{9} \mathrm{Be}$. For ${ }^{12} \mathrm{C}$, together with the Hoyle state, the contribution from the $2^{+}$ resonance is also included. When available, the experimental energy and width of the resonances are used as input for the calculation. Otherwise, like the $2^{+}$resonance in ${ }^{12} \mathrm{C}$, a theoretical estimate is employed. The required inelastic cross sections for $\alpha$, neutron, or proton scattering on ${ }^{9} \mathrm{Be}$ and ${ }^{12} \mathrm{C}$ are also constructed to reproduce the available experimental data.

As a general result, we have found that the two possible reaction mechanisms do not produce very different reaction rates, neither for ${ }^{9} \mathrm{Be}$ nor ${ }^{12} \mathrm{C}$, at intermediate and high temperatures, although the sequential process is always producing a higher rate at high temperatures. However, at very low temperatures, the rate is highly sensitive to both, the width of the resonances and the capture mechanism. In particular, a direct capture mechanism can produce reaction rates about seven or eight orders of magnitude higher than for the sequential mechanism.

Compared to the radiative production rates, we have found that the particle induced recombination can become dominant for mass densities in the vicinity of $10^{6}$ or $10^{7} \mathrm{~g} / \mathrm{cm}^{3}$ for the case of ${ }^{9} \mathrm{Be}$. This result is consistent with previous estimates. For ${ }^{12} \mathrm{C}$, the required mass density appears to be about two orders of magnitude smaller, in the vicinity of $10^{4}$ or $10^{5} \mathrm{~g} / \mathrm{cm}^{3}$.

Let us finish by emphasizing that the results obtained in this work are based on the reliability of the experimental information that permits to construct reliable resonant and nonresonant cross sections. In fact, a very detailed and accurate experimental information of the $\sigma_{A d}$ cross sections, especially at very small energies, would be enough to obtain accurate values for the reaction rates through Eq. (1). However, since these experimental data are very scarce, and at very small energies even very unlikely, we believe the analysis developed in this work can be a relevant tool providing the input in future astrophysical abundance calculations, where the important effects of nuclear four-body recombination are included. We would be pleased and also not surprised, if significant impact is encountered. However, this obvious perspective requires specialists for reliable implementation, and we therefore leave such application for future work.

\section{ACKNOWLEDGMENTS}

This work has been partially supported by the Spanish Ministerio de Economía y Competitividad and European Union FEDER funds under Contract No. PGC2018-093636B-I00. 
[1] A. Aprahamian, K. Langanke, and M. Wiescher, Prog. Part. Nucl. Phys. 54, 535 (2005).

[2] C. Angulo et al., Nucl. Phys. A 656, 3 (1999).

[3] K. Nomoto, F.-K. Thielemann, and S. Miyaji, Astron. Astrophys. 149, 239 (1985).

[4] K. Langanke, M. Wiescher, and F.-K. Thielemann, Z. Phys. A 324, 147 (1986).

[5] E. Garrido, R. de Diego, D. V. Fedorov, and A. S. Jensen, Eur. Phys. J. A 47, 102 (2011).

[6] J. W. Truran and B. Z. Kozlovsky, Astrophys. J. 158, 1021 (1969).

[7] J. F. Morgan and D. C. Weisser, Nucl. Phys. A 151, 561 (1970).

[8] C. N. Davids and T. I. Bonner, Astrophys. J. 166, 405 (1971).

[9] R. de Diego, E. Garrido, D. V. Fedorov, and A. S. Jensen, J. Phys. G: Nucl. Part. Phys. 37, 115105 (2010).

[10] R. de Diego, E. Garrido, D. V. Fedorov, and A. S. Jensen, Eur. Phys. J. A 50, 93 (2014).

[11] M. Beard, S. M. Austin, and R. Cyburt, Phys. Rev. Lett. 119, 112701 (2017).
[12] W. A. Fowler, G. R. Caughlan, and B. A. Zimmerman, Annu. Rev. Astron. Astrophys. 5, 525 (1967).

[13] E. Garrido, A. Kievsky, and M. Viviani, Phys. Rev. C 90, 014607 (2014).

[14] E. Garrido, Few-Body Syst. 59, 17 (2018).

[15] E. Garrido, D. V. Fedorov, A. S. Jensen, and H. O. U. Fynbo, Nucl. Phys. A 748, 27 (2005).

[16] D. R. Tilley, J. H. Kelley, J. L. Godwin, D. J. Millener, J. Purcell, C. G. Sheu, and H. R. Weller, Nucl. Phys. A 745, 155 (2004).

[17] D. R. Tilley, C. M. Cheves, J. L. Godwin, G. M. Hale, H. M. Hofmann, J. H. Kelley, C. G. Sheu, and H. R. Weller, Nucl. Phys. A 708, 3 (2002).

[18] J. H. Kelley, J. E. Purcell, and C. G. Sheu, Nucl. Phys. A 968, 71 (2016).

[19] J. H. Gibbons and R. L. Macklin, Phys. Rev. 114, 571 (1959).

[20] J. H. Gibbons and R. L. Macklin, Phys. Rev. 137, B1508 (1965).

[21] N. Olsson, B. Trostell, and E. Ramström, Phys. Med. Biol. 34, 909 (1989). 